\title{
Risikoadjustierung von Ergebnissen - Vorgehensweisen und Gefahren
}

\section{Hintergrund \\ $\nabla$}

Zentrales Ziel einer externen Qualitätssicherung ist die Vergleichbarkeit der erhaltenen Klinikergebnisse. Um dies zu gewährleisten werden seit jeher risikoadjustierte Analyseverfahren gefordert, wobei allgemein anerkannt ist, dass eine vollständige Risikoadjustierung nach allen möglichen Störfaktoren nicht durchführbar ist.

Bei der Verwendung von Routinedaten zur vergleichenden Analyse der Routinedaten wurde bislang oft berichtet, dass insbesondere relevante Informationen für eine Risikoadjustierung nicht zur Verfügung stehen. Allerdings zeigen einige hochrangig publizierte jüngere Arbeiten zum Thema durchaus vergleichbare Ergebnisse beim Vergleich der Prognosefähigkeit von Routinedaten und klinischen Registerdaten mit Bezug auf Indikatoren der Ergebnisqualität [1,2].

Im Folgenden wird dargestellt, wie bei der Implementierung von Risikoadjustierungsverfahren im Projekt „Qualitätssicherung mit Routinedaten“ (QSR) vorgegangen wurde und an welchen Möglichkeiten der Weiterentwicklung der Risikoadjustierung aktuell gearbeitet wird.

\section{Material und Methoden}

QSR basiert auf bundesweiten Krankenhausabrechnungsdaten nach §301 SGBV und auf anonymisierten Versichertenstammdaten. Zur Risikoadjustierung von tracerspezifischen Qualitätsindikatoren werden derzeit Alter und Geschlecht, aber auch relevante, bei Aufnahme mutmaßlich bereits existente Diagnosen, verwendet. Risikoadjustierte Kennzahlen (Erwartete Werte, SMRs und zugehörige Konfidenzintervalle) werden mit Hilfe von logistischen Regressionsgleichungen geschätzt. Risikoadjustierungsvariablen mit nicht plausiblen Ergebnissen wurden dabei ausgeschlossen. Die Diskriminationsfähigkeit der Modelle wurde mittels „Receiver-Operator-Characteristic-Analysen“ (c-Statistiken) untersucht. Daneben wurden die Einflussvariablen auf mögliche Multikollinearität untersucht.

\section{Diskussion \\ $\checkmark$}

Bei der Betrachtung des Modellfits sollte allerdings beachtet werden, dass eine bessere c-Statistik keinesfalls mit einer überlegenen Risikoadjustierung gleichzusetzen ist. Dies setzt eine ho- mogene Interpretation und Dokumentation der verwendeten Risikofaktoren voraus. Ist dies nicht der Fall kann ggf. eine ausführlichere Risikoadjustierung ebenfalls mit einem verzerrten Vergleich zwischen Krankenhäusern einhergehen [3].

\section{Schlussfolgerung}

Aktuelle Analysen zur Risikoadjustierung untersuchen die vorhandenen Routinedaten auf weitere relevante Risikoadjustierungsvariablen. Gleichzeitig wird eine Prüfung auf Homogenität der verwendeten Risikoadjustierungsvariablen durchgeführt.

Autorenerklärung: Der Autor arbeitet für das WIdO und den AOK-Bundesverband am Projekt „Qualitätssicherung der stationären Versorgung mit Routinedaten“.

\section{Literatur}

1 Aylin P, Bottle A, Majeed A. Use of administrative data or clinical databases as predictors of risk of death in hospital: comparison of models. BMJ 2007; 334: 1044-

2 Pine $M$, Jordan HS, Elixhauser A, Fry DE, Hoaglin DC, Jones B, Meinban R, Warner D, Gonzales J. Enhancement of Claims Data to Improve Risk of Hospital Mortality. JAMA 2007; 297: 71-76

3 Heller G, Schnell R. Hospital Mortality Risk Adjustment Using Claims Data. JAMA 2007; 297: 19831984

\section{G. Heller}

Qualitätsmanagement

Schlüsselwörter

Ergebnisqualität

Risikoadjustierung

$\checkmark$ Routinedaten

Medizinische Versorgung

Key words

outcomes of care

risk adjustment

administrative data

medical care

Institut

Wissenschaftliches Institut der AOK (WIdO)

Bibliografie

DOI 10.1055/s-0028-1085595 Dtsch Med Wochenschr 2008; 133: S151 - (c) Georg Thieme Verlag KG Stuttgart · New York . ISSN 0012-0472

Korrespondenz PD Dr. med. Günther Heller Wissenschaftliches Institut der AOK (WIdO)

Kortrijker Straße 1

D-53177 Bonn

Tel. +49 (228) 843121

eMail guenther.heller@

wido.bv.aok.de 